\title{
Antibiotic use in early childhood and risk of obesity: longitudinal analysis of a national cohort
}

\author{
Dervla Kelly $^{1}$ (D) Alan Kelly $^{1} \cdot$ Tom O'Dowd $^{1} \cdot$ Catherine B. Hayes $^{1}$
}

Received: 20 June 2018 / Accepted: 20 December 2018 / Published online: 12 January 2019

(c) Children's Hospital, Zhejiang University School of Medicine 2019

\begin{abstract}
Background Taking oral antibiotics during childhood has been linked with an increased risk of childhood obesity. This study assessed any potential association in number of courses of antibiotics taken between 2-3 and 4-5 years of age and body mass trajectory up to age 5 .

Methods The study was a secondary analysis of 8186 children and their parents from the infant cohort of the Irish National Longitudinal Study of Children. Antibiotic use was measured by parental recall between ages 2-3 and 4-5. Longitudinal models described the relationship between antibiotic exposure and body mass index (BMI) standard deviation scores and binary outcomes, and examined interactions between covariates, which included socioeconomic status, diet assessed by food frequency questionnaires and maternal BMI.

Results Any antibiotic usage between 2 and 3 years did not predict risk of overweight or obesity at age 5. Four or more courses of antibiotics between 2 and 3 years were independently associated with obesity at age 5 (odds ratio 1.6, 95\% confidence interval 1.11-2.31). Effect size was modest (coefficient +0.09 body mass SD units, standard error 0.04, $P=0.037$ ). Maternal BMI modified the relationship: $\geq 4$ courses of antibiotics between 2 and 3 years were associated with a +0.12 body mass SD units increase in weight at age 5 among children of normal-weight mothers $(P=0.035)$, but not in children of overweight mothers.

Conclusions Number of antibiotic courses, rather than antibiotic use, may be an important factor in any link between early antibiotic exposure and subsequent childhood obesity. Research is needed to confirm differential effects on babies of normal versus overweight/obese mothers independent of socioeconomic factors.
\end{abstract}

Keywords Antibiotics · Body mass index · Early childhood · Infants · Obesity

\section{Introduction}

Childhood obesity remains a major public health concern in western countries, including Ireland. It is a strong predictor of adult obesity [1]. Obesity is now recognized as a clinical condition [2] affecting all body systems and a major

Alan Kelly: RIP, 5th October 2015. Dublin, Ireland.

Electronic supplementary material The online version of this article (https://doi.org/10.1007/s12519-018-00223-1) contains supplementary material, which is available to authorized users.

Dervla Kelly

dkelly23@tcd.ie

1 Department of Public Health and Primary Care, Trinity College Dublin, Russell Center, Tallaght Cross, Dublin 24, Ireland contributor to chronic diseases including type 2 diabetes mellitus, cardiovascular disease and cancer morbidity and mortality [3-5]. Quality of life may be severely impaired as evidenced by psychological complications such as low self-esteem and depression [6, 7]. Obesity is a multifactorial condition and antibiotic consumption in childhood has been suggested as a potential contributing factor.

Antibiotic prescribing is common in young children especially for respiratory infections. Experimental evidence has shown that antibiotics disrupt the gut microbiota and these changes could disrupt metabolic processes. It is, therefore, plausible that agents modulating the microbiota, such as antibiotics, may affect body weight $[8,9]$ and there is concern that early childhood is a particularly vulnerable period when such effects may have long-term consequences [10].

The degree of influence of antibiotics on metabolism depends in theory on two determinants: (1) frequency of 
antibiotic exposure and (2) personal factors. While antibiotic dose is usually well defined, personal characteristics and behaviors are highly variable and difficult to capture (e.g., socioeconomic factors, diet, physical activity) [11]. Several epidemiological studies have suggested a positive relationship between childhood antibiotic use and obesity development [12-18]. A recent meta-analysis has found that azithromycin given to children with pulmonary disorders results in weight gain [19]. In contrast to the studies above, a large US cohort study by Gerber et al. found that antibiotic exposure during the first 6 months after birth was not significantly associated with weight gain trajectories from 0 to 8 years of age [20].

Four systematic reviews have been done to date, with variable findings. One concluded antibiotic exposure in early life significantly increased risk of childhood obesity [relative risk 1.21, 95\% confidence interval (CI) 1.13-1.30, $P<0.001$ ] [21], while a second found no consistent and conclusive evidence of associations between early life antibiotic use and later child body mass [22]. The other two reviews focused on antibiotic exposure in infants ( $<24$ months) and found that it was associated with a small increase in odds of childhood overweight or obesity in some children [23, 24].

The number of courses of antibiotics required to result in a lasting, meaningful impact on body mass has not yet been determined. In addition, it is unclear if antibiotic exposure during early childhood, post 24 months of age, is also disruptive to gut function. It is also unknown if the effect is sustained or reversible over time or whether multiple exposures during a particular period have a progressive effect. The aim of our study was to investigate any association between antibiotic use in early childhood and the trajectory of obesity from 9 months to 5 years in an Irish population, independent of known risk factors for obesity.

\section{Methods}

\section{Study design}

The study is a secondary analysis of a subset of the infant cohort of the Irish National Longitudinal Study of Children consisting of 8186 children. Variables with information on antibiotic exposure, body mass, socio-demographic variables and birthweight were extracted from the 9-month, 3and 5-year surveys.

\section{Study design and data collection of Irish National Longitudinal Study of Children}

The Infant Cohort of the National Longitudinal Study of Children, Growing Up in Ireland (GUI) has 8712 children and their caregivers. Data collection for the GUI study consisted of interviewer-administered questionnaires with parents in their home. The detailed study design has been published elsewhere [25]. The GUI study design was approved by the Scientific and Policy Advisory Committee and Research Ethics Committee and the Office of the Minister for Children and Youth Affairs in Ireland. Specific ethical approval was not required for this study.

\section{Study population}

Seven infants were excluded from the original cohort as the primary caregiver was not the biological parent; 519 were excluded as they were missing birthweight measurement or follow-up interview at either 3 or 5 years, resulting in a study sample of 8186 .

\section{Antibiotic exposure assessment}

Measures of antibiotic use were not available in the 9-monthold data. The primary caregiver was asked at ages 3 and 5: "Has child received a course of antibiotics in the past 12 months?" and "In total, how many courses of antibiotics has child received in the past 12 months?". No data were collected on type of antibiotics used, doses or prescribing indications; therefore, it was not possible to determine which antibiotics may be driving any effect.

Antibiotic exposure variables used in the analysis were: (1) use of any antibiotics as a binary yes/no covariate; (2) use of antibiotics as a categorical covariate to consider whether the number of courses of antibiotic exposure affects outcome.

In the year prior to data collection, $5285(64.6 \%)$ of 3 -year olds had at least one course of antibiotics. 2434 (29.7\%) had one antibiotic course, 1348 (16.5\%) had two antibiotic courses, 708 (8.6\%) had three antibiotic courses and 801 (9.8\%) had $\geq 4$ antibiotic courses (Table 1). 4589 (56.1\%) of 5-year olds had had at least one antibiotic course during the preceding year. $2322(28.4 \%)$ had one course, $1130(13.8 \%)$ had two courses, 555 (6.8\%) had three courses and $582(7.1 \%)$ had $\geq 4$ antibiotic courses (Table 1).

\section{Body weight measurement}

The child's weight and height or length were measured at 9 months, 3 and 5 years. A Leicester portable height stick was used to measure height. Weight was measured using a Class III medically approved SECA 835 portable electronic scales. Length was measured at 9 months using a SECA 210 measuring mat. Birthweight of the child was reported by the mother at 9 months.

Body mass index (BMI) was calculated by dividing the body weight (in kilograms) by the height (length if $<2$ years) (in meters) squared $\left(\mathrm{BMI}=\right.$ weight $/$ height $\left.^{2}\right)$. 
Table 1 Cohort description of the National Longitudinal Study of Children in Ireland with data from interviews carried out at 9 months, 3 years and 5 years of age

\begin{tabular}{|c|c|c|c|}
\hline Variables & $9 \operatorname{mon}(n=8186)$ & 3 y $(n=8186)$ & $5 \mathrm{y}(n=8186)$ \\
\hline \multicolumn{4}{|l|}{ Gender, \% $(n)$} \\
\hline Male & $50.7(4147)$ & & \\
\hline Female & $49.3(4039)$ & & \\
\hline \multicolumn{4}{|l|}{ Ethnicity, \% (n) } \\
\hline Irish or any other white background & $94.5(7731)$ & & \\
\hline Asian or Asian Irish & $2.1(174)$ & & \\
\hline Black or Black Irish & $2.9(240)$ & & \\
\hline Other, including mixed background & $0.5(39)$ & & \\
\hline \multicolumn{4}{|l|}{ Birth weight $(\mathrm{g}), \%(n)$} \\
\hline$<3000$ & $16.7(1356)$ & & \\
\hline $3000-4000$ & $67.7(5480)$ & & \\
\hline$>4000$ & $15.6(1262)$ & & \\
\hline \multicolumn{4}{|l|}{ Breastfeeding, \% (n) } \\
\hline Yes & $61.8(5060)$ & & \\
\hline No & $38.2(3125)$ & & \\
\hline \multicolumn{4}{|l|}{ Chronic illness child, \% $(n)$} \\
\hline No & $97.0(7940)$ & $85.3(6982)$ & $83.1(6809)$ \\
\hline Yes & $3.0(246)$ & $14.7(1204)$ & $16.8(1376)$ \\
\hline \multicolumn{4}{|l|}{ Maternal BMI, \% (n) } \\
\hline Non-overweight & $60.8(4977)$ & $53.3(4363)$ & $54.6(4327)$ \\
\hline Overweight/obese & $39.2(3209)$ & $46.7(3823)$ & $43.8(3592)$ \\
\hline \multicolumn{4}{|l|}{ Maternal education, \% ( $n)$} \\
\hline Degree or higher & $38.1(3120)$ & $41.3(3379)$ & $39.8(3255)$ \\
\hline Technical/diploma & $33.8(2768)$ & $37.2(3044)$ & $42.3(3460)$ \\
\hline Leaving cert & $18.2(1489)$ & $13.6(1112)$ & $11.5(940)$ \\
\hline Up to lower secondary & $9.9(809)$ & $7.9(647)$ & $6.5(531)$ \\
\hline \multicolumn{4}{|l|}{ Social class, \% (n) } \\
\hline Professional & $19.7(1609)$ & $18.5(1511)$ & $18.3(1501)$ \\
\hline Managerial and technical & 32.9 (2697) & $33.1(2711)$ & 33.8 (2764) \\
\hline Non-manual & $16.4(1344)$ & $16.8(1373)$ & $16.6(1357)$ \\
\hline Skilled manual & $13(1067)$ & $13.71123)$ & $12.2(1083)$ \\
\hline Semi-skilled and unskilled & $8.4(685)$ & $9.2(756)$ & $9.4(772)$ \\
\hline Validly no social class & $9.1(746)$ & $8.1(661)$ & $7.8(642)$ \\
\hline All others gainfully occupied/unknown & $0.5(38)$ & $0.6(51)$ & $0.8(67)$ \\
\hline \multicolumn{4}{|l|}{ Maternal smoking, $\%(n)$} \\
\hline Never & $77.4(6334)$ & $74.9(6097)$ & $77.0(6227)$ \\
\hline Occasionally & $7.1(585)$ & $8.8(714)$ & $8.9(715)$ \\
\hline Daily & $15.5(1266)$ & $16.3(1326)$ & $14.1(1140)$ \\
\hline \multicolumn{4}{|l|}{ Free GP access, $\%(n)$} \\
\hline No & $73.8(6043)$ & $65.4(5356)$ & $59.8(4892)$ \\
\hline Yes & $26.2(2136)$ & $34.6(2828)$ & $40.2(3294)$ \\
\hline \multicolumn{4}{|c|}{ Number of courses of antibiotics in the preceding y, $\%(n)$} \\
\hline 0 & & $35.4(2901)$ & $43.9(3592)$ \\
\hline 1 & & $29.7(2434)$ & $28.4(2322)$ \\
\hline 2 & & $16.5(1348)$ & $13.8(1130)$ \\
\hline 3 & & $8.6(708)$ & $6.8(555)$ \\
\hline $4+$ & & $9.8(801)$ & $7.1(582)$ \\
\hline \multicolumn{4}{|l|}{ Child's weight, \% ( $n$ ) } \\
\hline Normal & $72.2(5912)$ & $68.1(5580)$ & $83.8(6863)$ \\
\hline Overweight & $17.3(1413)$ & $19.2(1572)$ & $10.5(853)$ \\
\hline Obese & $10.5(861)$ & $12.7(1034)$ & $5.7(470)$ \\
\hline
\end{tabular}

$B M I$ body mass index, GP general practitioner 
Standard deviation (SD) scores of BMI (BMI SD scores) were calculated for the sample based on the UK-WHO dataset using "LmsGrowth", a Microsoft Excel add-in designed for use with growth chart references which provide age- and gender-specific cut offs [26]. Based on BMI SD scores, a sample population can be classified as underweight, normal, overweight and obese depending on how much they deviate from the population norm. The following BMI percentile cuts offs were applied: $\geq 91$ st percentile for overweight and $\geq 98$ th percentile for obesity.

The study outcome measurements were: (1) body mass as a logistic variable (obese- vs non-obese-dependent variable); (2) BMI SD score as a longitudinal linear dependent variable (longitudinal BMI SD score as a linear dependent variable) at 3 years and 5 years of age.

Prevalence of overweight was $20.4 \%$ (1748) at 5 years, with girls more likely to be overweight compared to boys [girls: 22.6\% (957); boys: 18.2\% (791)] (Table 1).

\section{Covariate assessment}

The following were included as potential confounders/effect modifiers in the analyses and were extracted from the surveys as described below.

Gender of study child, birthweight, gestational age and length of time of breastfeeding was recorded at 9 months.

Information on study child's diet, physical activity and health was obtained from age-5 questionnaire. A validated food frequency questionnaire measured in-depth dietary pattern data [27]. An estimate of the study child's daily calorie consumption was calculated from the food frequency information using the calories content from typical ageadjusted children's portion sizes [28] and nutrient values based on the McCance and Widdowson Food Composition Tables [29].

Other questionnaire data extracted at the age-5 survey included: socio-demographic information (social class, mother's age, maternal education, free general practitioner access, household income, household type, ethnicity), lifestyle characteristics [cigarette smoking, crèche (daycare) attendance] and measured maternal height and weight.

Table 1 summarizes the demographic and socioeconomic details. Half of the cohort, 50.7\% (4147) were males. The majority of the sample was "Irish or any other white background" $(94.5 \%, n=7731)$.

\section{Data analysis}

A cross-sectional description of the cohort was prepared from data collected at 9 months, 3 and 5 years of age. Longitudinal mixed models examined the relationship between antibiotic exposure and the repeated measures of BMI over time. Differences between exposed and unexposed subjects were tested at each of the three time points, with random effects used for subjects to account for individual patterns of weight change. Analyses were carried out using the "Ime4" package in $\mathrm{R}[30]$.

Four sets of longitudinal mixed models were developed using both outcome measurements: (1) obese vs non-obese; (2) longitudinal BMI SD score. The analyses compared body mass trajectory to four different antibiotic exposures: (1) any antibiotic use from 2 to 3 years; (2) number of courses of antibiotics taken between 2 and 3 years; (3) number of courses of antibiotics taken between 4 and 5 years and (4) cumulative antibiotic exposure from 2 to 3 years and 4 to 5 years. The final stage of the analysis involved fitting models with interaction terms (antibiotic use: gender) and stratified longitudinal mixed modeling by gender, having been breastfed, maternal BMI and maternal smoking to examine any interaction between covariates.

The results reported coefficients, standard errors and $P$ values for linear multilevel models or odds ratios (ORs) and 95\% CIs for binomial multilevel models. Statistically significant $P$ values were attained at $\alpha=0.05$. We plotted the mixed model results for number of courses of antibiotics used at $2-3$ years and body mass trajectory from 9 months to 5 years (coefficients and $95 \%$ CIs using coefplot 2 ) to quickly visualize the point estimates and measures of uncertainty of the fitted statistical models [31].

\section{Results}

\section{Longitudinal analysis of the association between any antibiotic exposure (yes or no) and body mass across early childhood ( 9 months to 5 years)}

In an unadjusted cross-sectional model, any antibiotic use at age 3 was statistically associated with a substantial increase in BMI SD scores [coefficient + 0.08 BMI SD units, standard error (SE) $0.03, P=0.001$ ]. In the logistic mixed model using categories obese vs non-obese as the outcome, any antibiotic use was not associated with being obese during early childhood, adjusting for other known predictors (OR 1.07, 95\% CI 0.75-1.52) (Table 2). Being obese was independently significantly associated with four predictors: Black or Black Irish ethnicity (OR 3.44, 95\% CI 1.25-9.47), higher birthweight (OR 1.95, 95\% CI 1.46-2.61), lower maternal education (OR 2.52, 95\% CI 1.34-4.72) and maternal BMI of $\geq 25$ (OR $1.43,95 \%$ CI $1.02-2.61)$. 
Table 2 Longitudinal mixed models of the association between the number of courses of antibiotics use at 2-3 years and/or 4-5 years and body mass trajectory from 9 months to 5 years adjusting for known confounders ${ }^{\mathrm{a}}$

\begin{tabular}{|c|c|c|c|c|c|c|}
\hline \multirow[t]{2}{*}{ Outcome variable } & \multicolumn{3}{|c|}{ BMI SD-score } & \multicolumn{3}{|c|}{ Obese vs non-obese } \\
\hline & Coefficient & SE & $P$ value & OR & 95\% CI (lower limit) & $\begin{array}{l}95 \% \mathrm{CI} \\
\text { (upper } \\
\text { limit) }\end{array}$ \\
\hline \multicolumn{7}{|c|}{ Exposure 1: use of "any" antibiotics between 2 and 3 y } \\
\hline No & Reference & & & 1.00 & & \\
\hline Yes & 0.03 & 0.02 & 0.263 & 1.07 & 0.75 & 1.52 \\
\hline \multicolumn{7}{|c|}{ Exposure 2: number of courses of antibiotics between age 2 and 3 y } \\
\hline 0 & Reference & & & 1.00 & & \\
\hline 1 & 0.01 & 0.03 & 0.646 & 0.88 & 0.61 & 1.27 \\
\hline 2 & 0.03 & 0.03 & 0.423 & 0.95 & 0.66 & 1.38 \\
\hline 3 & 0.02 & 0.04 & 0.710 & 1.16 & 0.80 & 1.69 \\
\hline$\geq 4$ & 0.09 & 0.04 & $0.037 *$ & 1.60 & 1.11 & $2.31^{*}$ \\
\hline \multicolumn{7}{|c|}{ Exposure 3: number of courses of antibiotics between age 4 and $5 \mathrm{y}$} \\
\hline 0 & Reference & & & 1.00 & & \\
\hline 1 & 0.05 & 0.03 & 0.093 & 1.15 & 0.80 & 1.64 \\
\hline 2 & 0.05 & 0.04 & 0.203 & 1.04 & 0.65 & 1.67 \\
\hline 3 & 0.03 & 0.05 & 0.593 & 1.16 & 0.63 & 2.16 \\
\hline$\geq 4$ & 0.05 & 0.05 & 0.372 & 1.37 & 0.74 & 2.53 \\
\hline \multicolumn{7}{|c|}{ Exposure 4: number of courses of antibiotics between age 2-3 and 4-5 y } \\
\hline 0 & Reference & & & 1.00 & & \\
\hline 1 & 0.07 & 0.02 & $<0.001 *$ & 1.05 & 0.79 & 1.41 \\
\hline 2 & 0.13 & 0.02 & $<0.001^{*}$ & 1.13 & 0.79 & 1.62 \\
\hline 3 & 0.14 & 0.03 & $<0.001 *$ & 1.36 & 0.85 & 2.17 \\
\hline$\geq 4$ & 0.12 & 0.03 & $<0.001 *$ & 1.63 & 1.01 & $2.62 *$ \\
\hline
\end{tabular}

$B M I$ body mass index, $S D$ standard deviation, $S E$ standard error, $O R$ odds ratio, $C I$ confidence interval. $* P<0.05$. ${ }^{a}$ Adjusted for gender, attending crèche, being breastfed, food energy intake (kcal), level of exercise, having a chronic illness, maternal BMI, birthweight of child, social class of household, maternal smoking, maternal education and ethnicity

\section{Longitudinal analysis of the association between number of courses of antibiotics and body mass across early childhood (9 months to 5 years)}

In the logistic mixed model, using $\geq 4$ courses of antibiotics during the 12-month period from 2 to 3 years was significantly associated with being obese during early childhood, adjusting for other known predictors (OR 1.60, 95\% CI 1.11-2.31) (Table 2). In the multivariable model, being obese was not associated with a higher calorific diet (OR $1.71,95 \%$ CI $0.79-3.69$ ) or having chronic illness (OR $1.22,95 \%$ CI 0.77-1.94) (Supplementary Table 1).

Increased BMI SD score was moderately associated with taking $\geq 4$ courses of antibiotics during the 12-month period from 2 to 3 years, adjusting for other known predictors (coefficient +0.09 BMI SD units, SE 0.04, $P=0.037$ ) (Table 2); (unadjusted univariate model: coefficient +0.24 BMI SD units, SE 0.04, $P<0.001$ ).

In contrast, taking $\geq 4$ courses of antibiotics during the 12 -month period from 4 to 5 years was not significantly associated with being obese during early childhood, adjusting for other known predictors (OR 1.60, 95\% CI 1.11-2.31) (Table 2).

Cumulative exposure $\geq 4$ courses of antibiotics exposures from age 2 to 3 and age 4 to 5 showed the strongest association with increased risk of obesity at age 5 (coefficient $+0.12, P<0.001$ ), having adjusted for other known confounders (Table 2). In the multivariable model, no association was found between being obese and having with a higher calorific diet (OR 1.59, 95\% CI 0.60-4.23) or having chronic illness (OR 1.05, 95\% CI 0.59-1.89) (Supplementary Table 2).

\section{Interaction effects of covariates}

In a longitudinal mixed model fitted with a gender by antibiotic use interaction term as well as the covariates above, there was no statistically significant interaction found (gender: $4+$ courses of antibiotics interaction term: coefficient -0.109 BMI SD units, $P=0.303 ; 4+$ courses of antibiotics: coefficient 0.18 BMI SD units, $P=0.029)$. In the models stratified by gender, there was no evidence that gender 
modified the association between antibiotic use and obesity ( $4+$ courses of antibiotics: coefficient 0.06 BMI SD units, $P=0.329$ ).

In the models stratified by breastfeeding, there was a nonstatistically significant trend of increasing BMI SD-score with increasing antibiotic use among those infants whose were breastfed $(4+$ courses of antibiotics: coefficient 0.12 BMI SD units, $P=0.090$ ).

There was a non-statistically significant trend of increasing BMI SD score with increasing antibiotic exposure among those infants whose parents did not smoke and had taken four or more courses of antibiotics $(4+$ courses of antibiotics: coefficient 0.09 BMI SD units, $P=0.054$ ).

There was no evidence of having a chronic illness modified the association between antibiotic use and obesity in the stratified models $(4+$ courses $\&$ having a chronic illness: $P=0.153 ; 4+$ courses and not having a chronic illness: $P=0.128)$.

In a longitudinal mixed model fitted with a maternal weight category by antibiotic use interaction term as well as the covariates above, there was no statistically significant interaction found (gender: $4+$ courses of antibiotics interaction term: coefficient -0.030 BMI SD units, $P=0.774$; $4+$ courses of antibiotics: coefficient 0.13 BMI SD units, $P=0.082)$. When the interaction between maternal weight and the antibiotic-obesity association was examined in stratified analysis, interaction between maternal weight, antibiotic exposure and obesity was observed. Children of normal-weight mothers exposed to $\geq 4$ courses of antibiotics between 2 and 3 years showed an increase of +0.12 BMI SD units $(P=0.035)$ compared to children of overweight and obese mothers (coefficient $+0.06, P=0.367$ ) .

\section{Discussion}

In this study, we set out to examine any effect of antibiotic use after the age of 2 years on BMI in childhood in an Irish longitudinal cohort study. Our results demonstrated that the only high levels of antibiotic use of $\geq 4$ courses of antibiotics between 2 and 3 years were associated with an increased body mass trajectory from 9 months to 5 years. Pooled data from two study waves strengthened the association. No association was detected at less than four courses or in crosssectional analysis of antibiotic exposure between ages 4 and 5. These findings highlight the importance of frequency of antibiotic use and age at administration in the detection of antibiotic-related weight changes. This would suggest that repeated antibiotic exposure may impair recovery of microbiota and metabolic functioning in young children and that microbiota recovery is possible with less frequent antibiotic exposure.
Our findings demonstrated a modest effect size of weight increase following antibiotic exposure. Translated into weight increments for the average 5 years old, a +0.09 SD unit increase in body mass corresponds to a $250-\mathrm{g}$ increase in weight. A comparable effect size was demonstrated by Schwartz et al. [32] (1 course: +0.01 BMI SD units, $P=0.009$; $4-6$ courses: +0.05 BMI SD units, $P$ value $=0.012$ ). Any effect of antibiotics on weight is unlikely to have significant impact at an individual level. However, the impact at a population level may be more significant.

Three studies found no or inconsistent risk of obesity with antibiotic use during childhood [20,33,34]. The differences in findings across studies are likely due, in part, to differences in the age of participants and intervals between antibiotic use and weight measurements, including self-report of child's weight measurements [33,34] and cross-sectional designs [34]. Furthermore, it has been suggested that gut microbiota may be less susceptible to alteration by antibiotics as it matures between birth and age 3 [35]. As we have no information available on antibiotic use prior to 2 years of age, it is also possible that the high antibiotic use observed in early childhood may be a persistent pattern from infancy that has biased our results.

The finding that children of normal-weight mothers exposed to $\geq 4$ courses of antibiotics between 2 and 3 years had an increased risk of obesity at age 5 compared to children of mothers with a BMI $>25$ was similar to two studies $[14,33]$. One hypothesis is that normal and overweight mothers have different gut microbial composition that is passed on to infants during delivery, predisposing the former to greater microbiota disruption with antibiotics $[33,36,37]$. Another possibility is that risk factors for obesity associated with maternal obesity may mask any relationship between antibiotics and obesity [38].

Obesity is a multifactorial condition. Many factors such as duration of breastfeeding, exercise, and health status have been shown to contribute to the risk of developing obesity. While these covariates did not appear to be statistically associated with changes in the antibiotic-obesity relationship in the population, it may suggest that antibiotics contribute to the risk of obesity via different biological mechanisms and/ or the antibiotic-obesity relationship does not follow typical socioeconomic patterning of health issues.

The main strength of the GUI cohort is that repeated objective measurements of weight and height were collected from study child and mother. Prior studies of antibiotic use and BMI were often limited by lack of follow-up weight measurements. Another advantage GUI is the wide range of available information on confounding social and environmental variables collected.

Notwithstanding these strengths, our study has some significant limitations. The study survey instruments were not specifically designed for this study; therefore, some 
desirable pieces of information on antibiotic use such as type, dose and prescribing indication were not available. The GUI study did not record antibiotic usage at 9 months. Recall bias limits the maternal report of antibiotic use, chronic illness diagnosis, length of time breastfed and accuracy of reported smoking behaviours.

In addition, BMI SD scores were the only measures available that would allow comparison between ages, as no alternative measure of obesity is available for children under 2 years of age [39, 40]. Using age-adjusted SD score removes some of the within-person variation in body mass and its use in longitudinal studies has been criticized because of this [41]. Also, it should be noted that the reference charts used in obesity studies change the rate of obesity detected across studies, with the UK-WHO reference charts used here giving higher rates of obesity compared with other cut-offs.

The persistent though modest association between high levels of antibiotic use and obesity, independent of predictors such as birthweight and maternal weight, is unlikely to be spurious. Additional studies addressing the biological mechanism behind the effect of antibiotic exposure and subsequent obesity would be a logical next step for research, including examination of the changes in bacterial gut profile associated with different types and doses of antibiotics. For clinical practice, deployment of new approaches in relation to prescribing practices based on the risk of obesity would be premature as there is insufficient evidence to infer that antibiotics cause obesity or are unsafe for children.

In conclusion, we found that high levels of antibiotic exposure of $\geq 4$ courses of antibiotics between ages 2 and 3 were associated with obesity at age 5 . Given the inconsistency of the effect between age $2-3$ and age $4-5$, it is likely the association is more complex than postulated in studies to date. The impact of antibiotics on metabolic function was evident after repeated courses of treatment suggesting that recovery is possible with less frequent antibiotic exposure. Given the current epidemic of childhood obesity and widespread use of antibiotics, further research is needed with a focus on detailed information on the types of antibiotics taken and biological sampling of gut bacteria before and after antibiotic use.

Author contributions DK contributed to study design, data analysis, and manuscript writing and review. AK contributed to study design and data analysis. TOD contributed to study design, manuscript writing, and manuscript review. $\mathrm{CH}$ contributed to study design, data analysis, and manuscript writing and review. All authors approved the final version of the manuscript.

Funding The National Study Longitudinal of Childhood-Growing Up in Ireland is funded by the Department of Children and Youth Affairs in Ireland. DK was funded by a stipend from Trinity College Dublin.

\section{Compliance with ethical standards}

Ethical approval This study was approved by a specially-convened Research Ethics Committee of The National Study Longitudinal of Childhood-Growing Up in Ireland.

Conflict of interest No financial or nonfinancial benefits have been received or will be received from any party related directly or indirectly to the subject of this article.

\section{References}

1. Reilly JJ, Kelly J. Long-term impact of overweight and obesity in childhood and adolescence on morbidity and premature mortality in adulthood: systematic review. Int J Obes (Lond). 2011;35:891-8.

2. Allison DB, Downey M, Atkinson RL, Billington CJ, Bray GA, Eckel RH, et al. Obesity as a disease: a white paper on evidence and arguments commissioned by the Council of the Obesity Society. Obesity (Silver Spring). 2008;16:1161-77.

3. Lloyd LJ, Langley-Evans SC, McMullen S. Childhood obesity and adult cardiovascular disease risk: a systematic review. Int J Obes (Lond). 2010;34:18-28.

4. Lauby-Secretan B, Scoccianti C, Loomis D, Grosse Y, Bianchini F, Straif K, et al. Body fatness and cancer-viewpoint of the IARC Working Group. N Engl J Med. 2016;375:794-8.

5. Yajnik CS. The lifecycle effects of nutrition and body size on adult adiposity, diabetes and cardiovascular disease. Obes Rev. 2002;3:217-24.

6. McLeod GF, Fergusson DM, John Horwood L, Carter FA. Adiposity and psychosocial outcomes at ages 30 and 35 . Soc Psychiatry Psychiatr Epidemiol. 2016;51:309-18.

7. Chaiton M, Sabiston C, O'Loughlin J, McGrath JJ, Maximova $\mathrm{K}$, Lambert M. A structural equation model relating adiposity, psychosocial indicators of body image and depressive symptoms among adolescents. Int J Obes (Lond). 2009;33:588-96.

8. Reinhardt C, Reigstad CS, Backhed F. Intestinal microbiota during infancy and its implications for obesity. J Pediatr Gastroenterol Nutr. 2009;48:249-56.

9. Musso G, Gambino R, Cassader M. Obesity, diabetes, and gut microbiota: the hygiene hypothesis expanded? Diabetes Care. 2010;33:2277-84.

10. Backhed F, Roswall J, Peng Y, Feng Q, Jia H, Kovatcheva-Datchary $\mathrm{P}$, et al. Dynamics and stabilization of the human gut microbiome during the first year of life. Cell Host Microbe. 2015;17:852.

11. Anglemyer A, Horvath HT, Bero L. Healthcare outcomes assessed with observational study designs compared with those assessed in randomized trials. Cochrane Database Syst Rev. 2014;4:Mr000034.

12. Azad MB, Moossavi S, Owora A, Sepehri S. Early-life antibiotic exposure, gut microbiota development, and predisposition to obesity. Nestle Nutr Inst Workshop Ser. 2017;88:67-79.

13. Bailey LC, Forrest CB, Zhang P, Richards TM, Livshits A, DeRusso PA. Association of antibiotics in infancy with early childhood obesity. JAMA Pediatr. 2014;168:1063-9.

14. Trasande L, Blustein J, Liu M, Corwin E, Cox LM, Blaser MJ. Infant antibiotic exposures and early-life body mass. Int J Obes (Lond). 2013;37:16-23.

15. Scott FI, Horton DB, Mamtani R, Haynes K, Goldberg DS, Lee DY, et al. Administration of antibiotics to children before age 2 years increases risk for childhood obesity. Gastroenterology. 2016;151:120-9.e5. 
16. Saari A, Virta LJ, Sankilampi U, Dunkel L, Saxen H. Antibiotic exposure in infancy and risk of being overweight in the first 24 months of life. Pediatrics. 2015;135:617-26.

17. Poulsen MN, Pollak J, Bailey-Davis L, Hirsch AG, Glass TA, Schwartz BS. Associations of prenatal and childhood antibiotic use with child body mass index at age 3 years. Obesity (Silver Spring). 2017;25:438-44.

18. Li DK, Chen H, Ferber J, Odouli R. Infection and antibiotic use in infancy and risk of childhood obesity: a longitudinal birth cohort study. Lancet Diabetes Endocrinol. 2017;5:18-25.

19. Dror T, Dickstein Y, Dubourg G, Paul M. Microbiota manipulation for weight change. Microb Pathog. 2017;106:146-61.

20. Gerber JS, Bryan M, Ross RK, Daymont C, Parks EP, Localio AR, et al. Antibiotic exposure during the first 6 months of life and weight gain during childhood. JAMA. 2016;315:1258-65.

21. Shao X, Ding X, Wang B, Li L, An X, Yao Q, et al. Antibiotic exposure in early life increases risk of childhood obesity: a systematic review and meta-analysis. Front Endocrinol (Lausanne). 2017;8:170.

22. Partap U, Allcock SH, Parker E, Gurdasani D, Young EH, Sandhu MS. Association between early life antibiotic use and childhood overweight and obesity: a narrative review. Glob Health Epidemiol Genom. 2018;3:e18.

23. Miller SA, Wu RKS, Oremus M. The association between antibiotic use in infancy and childhood overweight or obesity: a systematic review and meta-analysis. Obes Rev. 2018;19:1463-75.

24. Rasmussen SH, Shrestha S, Bjerregaard LG, Angquist LH, Baker JL, Jess T, et al. Antibiotic exposure in early life and childhood overweight and obesity: a systematic review and meta-analysis. Diabetes Obes Metab. 2018;20:1508-14.

25. Quail A, Williams J, McCrory C, Murray A, Thornton M. Sample design and response in wave 1 of the Infant Cohort (at 9 months) of Growing Up In Ireland. Dublin: Office of the Minister for Children and Youth Affairs, Department of Health and Children; 2011.

26. Pan H, Cole TJ. LMSgrowth, a Microsoft Excel add-into access growth references based on the LMS method. Version 2.77. 11017 ed 2012. http://www.healthforchildren.co/uk/. Accessed 1 Nov 2015.

27. Kelleher CC, Viljoen K, Khalil H, Somerville R, O'Brien J, Shrivastava A, et al. Longitudinal follow-up of the relationship between dietary intake and growth and development in the lifeways cross-generation cohort study 2001-2013. Proc Nutr Soc. 2014;73:118-31.

28. Wrieden WL, Longbottom PJ, Adamson AJ, Ogston SA, Payne A, Haleem MA, et al. Estimation of typical food portion sizes for children of different ages in Great Britain. Br J Nutr. 2008;99:1344-53.
29. Food Standards Agency. McCance and Widdowson's the composition of foods. 7th ed. London: Royal Society of Chemistry; 2014.

30. Bates D, Mächler M, Bolker B, Walker S. Fitting linear mixedeffects models using lme4. arXiv preprint arXiv:1406.5823; 2014.

31. Bolker B, Su Y-S. Coefplot2: coefficient plots. R package version 0132; 2011.

32. Schwartz BS, Pollak J, Bailey-Davis L, Hirsch AG, Cosgrove SE, Nau C, et al. Antibiotic use and childhood body mass index trajectory. Int J Obes (Lond). 2015;40:615-21.

33. Ajslev TA, Andersen CS, Gamborg M, Sørensen TIA, Jess T. Childhood overweight after establishment of the gut microbiota: the role of delivery mode, pre-pregnancy weight and early administration of antibiotics. Int J Obes (Lond). 2011;35:522-9.

34. Murphy R, Stewart AW, Braithwaite I, Beasley R, Hancox RJ, Mitchell EA. Antibiotic treatment during infancy and increased body mass index in boys: an international cross-sectional study. Int J Obes (Lond). 2014;38:1115-9.

35. Langdon A, Crook N, Dantas G. The effects of antibiotics on the microbiome throughout development and alternative approaches for therapeutic modulation. Genome Med. 2016;8:39.

36. Collado MC, Isolauri E, Laitinen K, Salminen S. Distinct composition of gut microbiota during pregnancy in overweight and normal-weight women. Am J Clin Nutr. 2008;88:894-9.

37. Collado MC, Isolauri E, Laitinen K, Salminen S. Effect of mother's weight on infant's microbiota acquisition, composition, and activity during early infancy: a prospective follow-up study initiated in early pregnancy. Am J Clin Nutr. 2010;92:1023-30.

38. Linabery AM, Nahhas RW, Johnson W, Choh AC, Towne B, Odegaard AO, et al. Stronger influence of maternal than paternal obesity on infant and early childhood body mass index: the Fels Longitudinal Study. Pediatr Obes. 2013;8:159-69.

39. Cattaneo A, Monasta L, Stamatakis E, Lioret S, Castetbon K, Frenken F, et al. Overweight and obesity in infants and pre-school children in the European Union: a review of existing data. Obes Rev. 2010;11:389-98.

40. Roy SM, Chesi A, Mentch F, Xiao R, Chiavacci R, Mitchell JA, et al. Body mass index (BMI) trajectories in infancy differ by population ancestry and may presage disparities in early childhood obesity. J Clin Endocrinol Metab. 2015;100:1551-60.

41. Berkey CS, Colditz GA. Adiposity in adolescents: change in actual BMI works better than change in BMI $\mathrm{z}$ score for longitudinal studies. Ann Epidemiol. 2007;17:44-50.

Publisher's Note Springer Nature remains neutral with regard to jurisdictional claims in published maps and institutional affiliations. 